area, the white broad fibres seem to be well equipped largely for functioning anaerobically. On the other hand, the high mitochondrial content, together with the large store of fat, greater lipese activity, the presence of myoglobin and greater surface area afforded in the narrow fibres, suggests that they have a well-organized oxidative system. Further work towards a clearer understanding of the potentialities of these two distinet types of fibre and their mitochondria is in progress.

One of us (R. M. N.) is indebted to the Government of India for the award of a Senior Research Scholarship, which made this work possible.

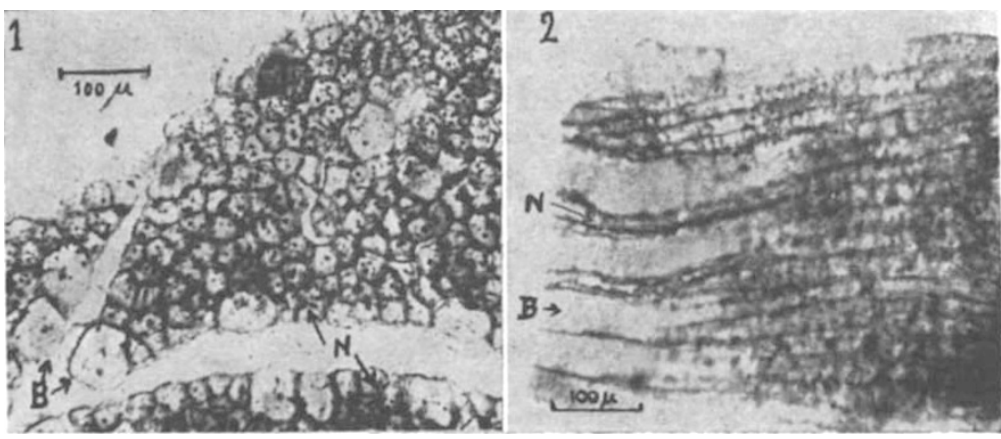
Photomicrographs of (1) a transverse and (2) an oblique section of the pectoralis
major muscle of the pigeon showing the disposition of lipase. $B$, broad flbres; $N$, narrow fibres

J. C. George

R. M. NAIK

Laboratories of Histochemistry and Animal Physiology, Department of Zoology, M.S. University of Baroda, Baroda, India. Nov. 7.

1 George, J. C., and Naik, R. M., Nature, [181, 709 (1958)].

2 George, J. C., and Scaria, K. S., Nature (see following communication). ${ }^{3}$ Jyoti, D., Ph.D. thesis, Baroda University, India (1956).

4 George, J. C., and Scaria, K. C., J. Anim. Morph. Physiol., 3, 91 (1956).

${ }^{5}$ Gray, P., "Microscopist's Formulary and Guide" (Blakiston, New York, 1957).

'Chappell, J. B., and Perry, S. V., Biochem. J., 55, 586 (1953).

'Paul, M. H., and Sterling, E., cited by Perry in Physiol. Rev., 36, 1 (1956).

\section{Histochemical Demonstration of Lipase Activity in the Pectoralis Major Muscle of the Pigeon}

OUR earlier observation that the pigeon breast muscle contains a lipase ${ }^{1}$ has led to the present attempt to demonstrate its presence in this muscle using histochemicel methods. The 'Tween' method of Gomori ${ }^{2}$ was chosen for the purpose. The sub. strate used was 'Tween 80' (Atlas). The incubation medium contained $2 \mathrm{ml}$. 10 per cent calcium chloride, $2 \mathrm{ml}$. 5 per cent 'Tween 80 ', $5 \mathrm{ml}$. bicarbonate buffer' of $p \mathbf{H ~ 8 . 4 , 4 0 ~ m l . ~ d i s t i l l e d ~ w a t e r ~ a n d ~ a ~ c r y s t a l ~ o f ~}$ thymol as preservative. The mixture was kept overnight in an oven at $40^{\circ} \mathrm{C}$. and the precipitate formed was filtered off before use. Sections were incubated for $10-12 \mathrm{hr}$. at $40^{\circ} \mathrm{C}$, which is the optimum temperature for this lipase ${ }^{1}$. Parallel experiments were run with sections of pancreas of the same animal for comparison and confirmation of the results. It was noted that acetone destroyed the lipsese, and experiments with paraffin sections of tissue fixed in acetone yielded negative results.

A very simple method of preparing sections was adopted and was found to be highly satisfactory. The tissue was cut out immediately after decapitating the animal and placed over ice in the deep freeze of a refrigerator; it was frozen hard in less than half an hour, and without removing it from the doep freeze it was trimmed to shape and thin sections cut with the aid of a cold, sharp razor blade and transferred to an albuminated slide by means of a cold needle. Very thin sections without any appreciable loss of the enzyme in them could thus be obtained. After drying at room temperature to ensure adhesion, the sections were fixed for $\mathrm{I} 6 \mathrm{hr}$. in cold $\left(4^{\circ} \mathrm{C}\right.$.) $6 \mathrm{per}$ cent formalin neutralized with phosphate. They were then washed in running water for about an hour, washed with distilled water and incubated in the above incubation medium. The rest of the procedure was the same as described by Pearse. . Since the fibres were stained brown after treatment with ammonium sulphide, the sections were differentiated by treatment with a few drops of hydrogen peroxide in a Coplin jar of 70 per cent alcohol for a few seconds. The sections were not counterstained. Sections which were boiled for $10 \mathrm{~min}$. were used as controls.

Fig. 1 shows the cross-section of a part of the pectoralis major muscle of the pigeon (Columba livia) in which the two types of fibres, a narrow and a broad variety, as described by George and Naik ${ }^{5,6}$, are clearly discernible. The dark spots in the photograph represent the brownish black precipitate of lead sulphide. It can be seen that the precipitate is abundant in the fat-loaded narrow fibres, whereas the glycogen-loaded broad fibres contain very little. Control sections do not show any precipitate. It is therefore inferred that in the pigeon breast muscle the lipase occurs mostly in the narrow fibres. How. ever, the occurrence of a few grains of the precipitate in the broad fibres is not convincing evidence that they are formed in the same fibres; study of a large number of sections indicates that these granules in the broad fibres are the result of diffusion of products of hydrolysis from the narrow fibres. Fig. 2, showing an oblique section of the muscle giving the longitudinal aspect of the two types of fibres, shows large areas of the broad fibres devoid of these precipitates. This supports the view that lipase is confined, by and large, to the narrow fibres, and very little, if any, occurs in the broad fibres. By contrast, in the pancreas, where lipase is known to be present in large amounts, the precipitate was found to be thickly dispersed throughout the section.

\section{J. C. GeORGE}

Histochemical Laboratory,

Department of Zoology,

M.S. University of Baroda,

Baroda, India. Sept. 9.

${ }^{1}$ George, J. C., and Scaria, K. S., J. Anim. Morph. Physiol., 3, 91 (1956)

2 Gomori, G., "Mieroscopic Histochemistry, Principles and Practice" (Univ. Chicago Press, Chicago, 1953).

${ }^{3}$ Sneath, P. H. A., cited by Pearse.

"Pearse, A. G., "Histochemistry, Theoretical and Applied" (J. and A. Churchill, London, 1954).

${ }^{5}$ George, J. C., and Naik, R. M., J. Anim. Morph. Physiol., 4, 23 (1957).

- George, J. C., and Naik, R. M., Nature [181, 709 (1958)]. 